\title{
Reflexões sobre o cuidado de enfermagem e a autonomia do ser humano na condição de idoso hospitalizado
}

\author{
Reflections on the care of nursing and the autonomy of the human being under \\ the condition of hospitalized elderly \\ Reflexiones sobre el cuidado de enfermería y la autonomía del ser humano \\ en la condición de anciano hospitalizado
}

\begin{abstract}
Marisa Basegio Carretta', Luiz Antonio Bettinelli', Alacoque Lorenzini Erdmann"
' Universidade de Passo Fundo, Programa de Mestrado em Envelhecimento Humano, Grupo de Estudos em Bioética e Cuidado Humano. Passo Fundo-RS, Brasil.

" Universidade Federal de Santa Catarina, Programa de Pós-Graduação em Enfermagem, Grupo de Estudos e Pesquisas em Administração e Gerência do Cuidado em Enfermagem e Saúde. Pesquisadora CNPq. Florianópolis-SC, Brasil.
\end{abstract}

Submissão: 15-01-2010 Aprovação: 13-12-2011

\section{RESUMO}

Com a aceleração do crescimento demográfico de idosos ocorre o aumento na prevalência de doenças crônicas, com a consequente necessidade de cuidados e a hospitalização. O presente artigo teve por objetivo refletir sobre o cuidado de enfermagem e a autonomia do ser humano na condição de idoso hospitalizado. Profissionais de enfermagem, por meio do cuidado de enfermagem no ambiente hospitalar, podem contribuir significativamente para a construção da autonomia e participação das pessoas idosas na tomada de decisão sobre suas necessidades de atenção ou cuidado à sua saúde. O resgate da ética numa perspectiva de envelhecimento digno perpassa por diversas discussões na sociedade que envelhece e, especificamente, entre os profissionais de saúde que atuam nas instituições hospitalares.

Descritores: Enfermagem; Idoso; Autonomia; Assistência hospitalar.

\section{ABSTRACT}

There has been an increase of chronic diseases with the subsequent need for care and hospitalization, due to the acceleration of the elderly demographic growth. The objective of the present paper is to reflect on the care of nursing and the autonomy of the human being under the condition of elderly hospitalization. Nursing professionals, caring in the hospital ambient, can contribute significantly for the construction of the elderly autonomy and participation on the decision making about their need for attention or health care. The ethics redeeming, under a worthy aging perspective, permeates several discussions in the aging society and, specifically, among health professionals who act in hospital institutions.

Key words: Nursing; Elderly; Autonomy; Hospital assistance.

\section{RESUMEN}

Con la aceleración del crecimiento demográfico de ancianos ocurre el aumento en la prevalencia de enfermedades crónicas, con la consecuente necesidad de cuidados y la hospitalización. El presente artículo tuvo por objetivo refletar sobre el cuidado de enfermería y la autonomía del ser humano en la condición de anciano hospitalizado. Profesionales de enfermería a través del cuidado de enfermería en el ambiente hospitalario pueden contribuir significativamente para la construcción de la autonomía y participación de las personas ancianas en la toma de decisiones sobre sus necesidades de atención o cuidado a su salud. El rescate de la ética en una perspectiva de envejecimiento digno pasa por diversas discusiones en la sociedad que envejece $y$, específicamente, entre los profesionales de salud que actúan en las instituciones hospitalarias.

Descriptores: Enfermería; Anciano; Autonomía; Asistencia hospitalaria. 


\section{ENVELHECIMENTO HUMANO E NECESSIDADE DE CUIDADO DE ENFERMAGEM HOSPITALAR}

O envelhecimento populacional global vem sendo apontado como um fenômeno já estimado há algumas décadas, entretanto a sociedade ainda revela dificuldades no manejo desta população crescente. Existem cerca de seiscentos miIhões de idosos vivendo no mundo. O montante desta população perfaz 8,6\% do total de habitantes nacionais. Também estimativas apontam um aumento de $15 \%$ para 2020 , os octogenários correspondem a $11 \%$ do total de idosos e a projeção para 2050 comporta 19\% nesta faixa etária. O mesmo censo vislumbra um expressivo aumento de octogenários: de $11 \%$ em 2000 para $19 \%$ em 2050). Esse dado representa praticamente o dobro de idosos na pirâmide populacional ${ }^{(1)}$.

Dados alertam que as doenças crônicas não transmissíveis (DCNT), em nível mundial, são responsáveis por 59\% dos óbitos e $46 \%$ do número total de doenças globais. Nos países em desenvolvimento o impacto produzido por essas doenças é acentuado, uma vez que a estrutura social em envelhecimento não se preparou para o enfrentamento comportamental e econômico de tal situação. No Rio Grande do Sul, a população em geral apresenta a chamada "polaridade epidemiológica", resultado do processo de industrialização e urbanização, de diminuição da mortalidade infantil, de mudanças de hábito e estilo de vida, com incorporação de fatores de risco comportamental e ocupacional. Essa polaridade traz como consequência um aumento dos custos da assistência à saúde decorrente de doenças cardiovasculares, neoplasias, doenças respiratórias crônicas e causas externas ${ }^{(2)}$.

Também nesse contexto, as DCNT estão relacionadas ao aumento da expectativa de vida das pessoas, com o qual há o crescimento de incapacidades e limitações em idosos. Assim, a prevenção dessas doenças e o cuidado à saúde de pessoas idosas dependentes representam novos desafios para os profissionais da saúde e as políticas públicas e sociais de atenção à saúde da população.

O envelhecimento humano não significa diretamente convívio com as DCNT e incapacidades funcionais, mas a incidência destas preocupa as pessoas envolvidas nesse processo. Assim, os idosos estarão mais suscetíveis às tais doenças, que poderão levá-los a incapacidades funcionais e à dependência. O comprometimento da capacidade funcional tem implicações importantes para a família, comunidade, sistema de saúde e a vida do próprio idoso, uma vez que ocasiona maior vulnerabilidade e dependência, podendo contribuir para a diminuição da qualidade de vida ${ }^{(3)}$.

Desse modo, o idoso aponta como um dos principais usuários dos serviços de saúde no que tange à hospitalização(4). A internação hospitalar pode ser considerada um fator de desestruturação físico-emocional ao idoso, visto que ações e interações ocorrem num vínculo estabelecido por força situacional e estrutural. Os aspectos que suportam essa relação estão cercados, de um lado, por necessidades urgentes de atendimento e, de outro, de uma atividade profissional hierarquicamente determinada e dimensionada. Esse contato tende a ser muito próximo e complexo.
No final do século XVIII o hospital surgiu como ambiente terapêutico, pois anteriormente, este espaço era reservado aos desassistidos que se encontravam à margem da sociedade. $\mathrm{Na}$ vigência da Revolução Industrial, os profissionais da medicina passaram a utilizar este ambiente para procedimentos de diagnóstico e tratamento(5).

Com o engajamento de outras profissões, a função do hospital expandiu-se, integralizando em sua base administrativa funções médico-sociais, educacionais, de capacitação de recursos humanos e pesquisa em saúde ${ }^{(4)}$.

Essa estrutura, que objetiva recuperar a saúde do idoso, se constitui por normas, rotinas e regimentos padronizados, nem sempre flexíveis e com uma filosofia centrada na otimização das ações e resolutividade na recuperação da saúde. De outro modo, a complexidade assistencial hospitalar abarca padronizações que tendem a conectar o ser humano/idoso ao domínio da instituição de saúde em detrimento da personalização do cuidado, massificando os procedimentos e orientações com a finalidade de atender à demanda dos serviços.

O cenário de fronteira entre o ser humano/idoso internado e a estrutura administrativa hospitalar do fazer o trabalho terapêutico requer flexibilizar as práticas centradas no idoso/sujeito que originou todo esse processo. Nesse sentido, é oportuno questionar: Quem é este idoso de quem se está cuidando?

Esta pessoa não é qualquer coisa indefinida. É um rosto com olhar e fisionomia. Esse rosto torna impossível a indiferença. Ele me obriga a tomar posição porque fala. E em especial sendo um o rosto do empobrecido, marginalizado e excluído ${ }^{(6)}$.

O cotidiano das relações do cuidado nas instituições hospitalares, muitas vezes indiferente, mecânico e automatizado, não facilita a percepção do outro, tornando massificada a atuação profissional. Esses aspectos favorecem a despersonificação do ser humano, o que, por conseguinte, põe em risco a manutenção de sua dignidade.

O paternalismo profissional gerado no ambiente hospitalar oferece certa possibilidade de se tomar decisões pelos outros. A tomada de decisão acerca do processo da saúde do outro é um direito não concedido, porém executado rotineiramente. A única indagação acerca da tomada de decisão é sobre quem tem autoridade para a tomada de decisão por outra pessoa ${ }^{(7)}$.

A prática do cuidado de enfermagem em nível hospitalar vem acompanhada de posições específicas ocupadas por determinadas profissões na rede do cuidado ao idoso. Cada meta a ser atingida engloba a atividade racional e efetiva de cada componente destas posições. Protocolos rigorosos compartilham o atendimento humanizado pretendido, com ações facetadas e designadas uniformemente a todos os pacientes, logo, automatizadas. A racionalidade terapêutica, invariavelmente, ocupa seu espaço ordenando, fragmentando e despersonalizando as ações do cuidado.

O valor de uma vida é principalmente (ainda que não exclusivamente) o valor que ela tem para a pessoa que a possui. Partindo do pressuposto de que os profissionais da saúde trabalham com a vida do outro, surge a necessidade de reavaliar os limites das ações no espaço organizacional do cuidado e discutir a possibilidade de se ofertar esse cuidado de forma a 
respeitar a inclusão da autonomia do indivíduo que requer o atendimento hospitalar ${ }^{(8)}$.

A Política Nacional de Saúde da Pessoa Idosa (PNSPI) tem como objetivo assegurar os direitos sociais dessa parcela da população, promovendo a sua autonomia ${ }^{(9)}$. Esse idoso, quando hospitalizado tem sua autonomia relegada a um plano imperceptível, decorrente da hierarquização do cuidado e do biopoder estabelecido nas instituições. A situação de fragilidade inerente ao processo de internamento em instituições hospitalares retira o idoso de suas atividades de vida diárias cotidianas, além de reduzir drasticamente sua capacidade de decisão. Sendo o hospital altamente tecnológico, priorizam-se procedimentos e rotinas necessárias ao pleno funcionamento da instituição.

A interação com o ser humano/idoso é ainda pouco avaliada e parece natural que o cuidado seja de responsabilidade do profissional, o que o leva sentir-se autorizado a decidir, orientar, prover e administrar a vida do mesmo, pessoa idosa hospitalizada.

As perdas durante a hospitalização, como a possibilidade de manifestação da capacidade decisória/autonômica do paciente. Ao privar o idoso desta capacidade retira-se dele a responsabilidade pelos seus problemas de saúde, o que pode ter como consequência a responsabilização unilateral do sistema em torno do sujeito idoso hospitalizado.

Talvez os profissionais acreditem ter o poder de decisão sobre as relações de cuidado com os idosos hospitalizados, ou seja, tomar as decisões em nome deles. Porém, há de se ter um equacionamento do processo decisório delineador de uma situação que envolve uma mão de via dupla.

Atitudes estereotipadas com relação à dependência plena e total do idoso hospitalizado repercutem socialmente, uma vez que, retirada a sua autonomia, contribui-se para a determinação da incompetência do ser humano acometido e já fragilizado pela doença. Dessa forma, percorre-se a contramão do princípio da humanização, norteador de inúmeros questionamentos e debates atuais.

A possibilidade de promover a coparticipação do idoso na elaboração das rotinas que envolvem seu atendimento pode ser uma alternativa de avanço no maior respeito a autonomia e direito de tomada de decisão com e para os mesmos. Entender esse processo e investigar a expectativa do idoso nesse contexto possibilitará argumentos para uma mudança dos parâmetros na condução do cuidado hospitalar.

Mas será que o ser humano/idoso percebe a sua pouca autonomia? Será que isso o incomoda? Existe possibilidade de gerência para um cuidado mais autônomo? Quais são os riscos relativos e qual será a responsabilização das partes envolvidas? Refletir sobre essas inquietações torna-se importante para que os profissionais da saúde que atuam nas instituições hospitalares criem estratégias acerca do cuidado e do seu planejamento, possibilitando ao ser humano/idoso internado fazer escolhas sobre o cuidado a sua saúde/doença, exercendo assim sua autonomia e cidadania.

Assim aponta-se como principal questionamento: como os profissionais da enfermagem, através do cuidado de enfermagem, podem contribuir para a construção da autonomia do ser humano na condição de idoso hospitalizado? Desse modo, o presente artigo teve por objetivo refletir sobre cuidado de enfermagem e autonomia do ser humano na condição de idoso hospitalizado.

\section{AUTONOMIA DO IDOSO NO AMBIENTE HOSPITALAR}

Inúmeras são as possibilidades de discussões acerca da autonomia e dependência do idoso. Especificamente no ambiente hospitalar, a capacidade decisória do ser humano/ idoso pode encontrar-se cerceada por questões administrativas e burocráticas inerentes ao contexto institucional hospitalar. A complexidade técnica que permeia o cuidado neste domínio produz uma realidade diferente e nova ao ser humano. Ali a relação do cuidado acontece de forma mais assimétrica, pois a pessoa idosa, já fragilizada pela doença, sente-se talvez mais impotente diante da complexidade do ambiente hospitalar, o que enseja a sua dependência ao profissional.

A fragilidade do ser humano acometido por alguma doença, e dependente de outro, potencializa ainda mais a dificuldade de tomar decisões autônomas e deposita nos provedores do cuidado hospitalar a responsabilidade de ver esse sujeito como pessoa com necessidades, valores, experiências e intenções próprias.

A integração e a participação efetiva do ser humano/pessoa idosa hospitalizada em seu plano terapêutico revertem em mudanças sociais, estruturais e de relações de poder estabelecidas nas instituições de saúde a partir do momento da hospitalização. O idoso hospitalizado mantém suas características prévias, as quais necessitam ser respeitadas e preservadas; logo, é importante ver o ser humano como o sujeito de atenção do sistema, não o contrário. Dessa forma, o cuidado ao idoso hospitalizado, pode ocorrer norteado por relativa autonomia, desafiando as estruturas hospitalares a presenciar e viver um novo horizonte de atenção integral, no qual os princípios éticos alcancem uma dimensão mais ampla e efetiva, buscando a ampliação da qualidade do cuidado.

A autonomia sugere tomada de decisão deliberada ou mais livre, preservação da integridade e individualidade, baseada em aspirações, valores, crenças e objetivos particulares de cada ser. A oportunidade da manifestação da vontade e necessidade individual em nível hospitalar determina uma clara dissociação entre rotinas preestabelecidas e invariavelmente inflexíveis e a autonomia do paciente idoso. A hierarquização do cuidado e o empoderamento dos saberes em torno do processo saúde e doença, acrescidos às deliberações dos códigos de ética, cerceiam a tomada de decisão do idoso, favorecendo a sua passividade.

O envolvimento do conteúdo teórico conceitual com a prática da autonomia do idoso hospitalizado engloba verificar a possibilidade da utilização das capacidades e habilidades prévias inerentes ao ser idoso com o paternalismo profissional-social pertinente ao cuidado hospitalar.

O conflito estabelecido revela um antagonismo notável entre a teoria e a prática diária do cuidado hospitalar. A racionalidade da tomada de decisão competente posiciona-se, invariavelmente, num só lado do binômio paciente idoso/instituição hospitalar. A articulação dessa estrutura apresenta-se 
de forma a demonstrar dificuldades de integração que somente podem ser superadas com a diminuição do distanciamento dos sujeitos envolvidos no processo do cuidado.

Intermediações jurídicas e burocráticas necessitam ser estabelecidas a fim de possibilitar esta mudança conceitual e cultural, e rever práticas profissionais e relações de poder. Entende-se que a vulnerabilidade do paciente em questão pode induzir a um sentimento de perda de individualidade, muito comum nesse contexto.

A autonomia relaciona-se com a possibilidade da tomada de decisão. Para isso ocorrer é fundamental rever a relação que se estabelece entre os sujeitos envolvidos na ação. Na condição de contratante, o ser humano/paciente necessita ter claramente explicitada sua posição como pessoa reconhecida, possuidora de direitos sobre as decisões acerca de seu tratamento ${ }^{(8)}$.

Tal relação deve estar alicerçada no âmbito legal e de direitos humanos. Os limites deste alicerce estão envoltos pelo princípio da autonomia que se refere ao respeito devido aos direitos fundamentais do homem ${ }^{(10)}$. Incorporar a autonomia nesse contexto traz à luz a necessidade de revisão conceitual desse princípio.

A autonomia aqui referida não pode ser entendida como poder absoluto e ilimitado, uma vez que isso induziria à autossuficiência, a qual não encontra espaço nessa relação. Necessita haver uma compatibilidade entre o poder individual e os demais poderes que circundam o contexto da hospitalização da pessoa idosa.

Deliberar e escolher são entendimentos, significativos para a autonomia ${ }^{(8)}$. Ao deliberar, planejam-se meios adequados para atingir determinado fim, ao passo que escolher denota possibilidade de opção entre os caminhos oferecidos. Além disso, estabelece condições para a realização de uma opção autônoma: agir com intencionalidade, agir com conhecimento de causa e sem influências controladoras(11). Assim, problematiza-se a situação posta num ambiente hospitalar sistematicamente estruturado segundo rotinas e padrões preestabelecidos segundo a racionalidade profissional. Existe efetivamente a possibilidade da realização de uma opção autônoma neste ambiente?

Diante da relatividade da ação autônoma, deve-se levar em consideração o respeito ao direito e à vontade do outro. $\mathrm{O}$ princípio da autonomia pode ser renomeado por respeito à pessoa ou, ainda, por consentimento, de acordo com o autor e a época de referência ${ }^{(12)}$.

A autonomia da vontade é a constituição da vontade, pela qual ela é para si mesma uma lei-independentemente de como forem constituídos os objetos do querer. O princípio da autonomia é, pois, não escolher de outro modo, mas sim deste: que a máxima da escolha, no próprio querer, seja ao mesmo tempo incluída como lei universal ${ }^{13}$.

A autonomia é a competência da vontade humana, que deve poder se adequar a uma lei universal. A racionalidade da tomada de decisão consciente e aplicável universalmente sugere o respeito à autonomia como prerrogativa de um cuidado ético, estabelecido entre o profissional da saúde e o idoso devidamente orientado e informado sobre as intervenções de saúde a serem executadas durante seu internamento hospitalar ${ }^{(14)}$.
Redimensionando o conceito de autonomia emerge o princípio do consentimento, como estratégia moral e solidamente fundamentada por conceitos legais. Mais do que um trâmite burocrático, este princípio assegura autonomia, uma vez que só pode ocorrer após instrução e orientação adequadas. Por sua vez, os determinantes circunstanciais que asseguram o entendimento por parte dos envolvidos são relativos e nem sempre seguros.

O exercício pleno da autonomia na condição de ser humano idoso, fragilizado e dependente de cuidados de enfermagem, segue sendo um desafio a ser perseguido. Mas, quais são estes desafios?

\section{APONTANDO DESAFIOS}

A transição demográfica da humanidade vem alterando a realidade do cenário social, trazendo a longevidade como fator desencadeante de significativas mudanças estruturais. $\mathrm{O}$ ser humano envelhece, aumenta sua expectativa de vida e evidencia-se o surgimento de um maior número de doenças crônico-degenerativas impactantes para a estrutura social. Como enfrentar essa realidade?

Os cuidados com a saúde, eventualmente fragilizada, da pessoa idosa são permeados por dificuldades. As comorbidades se estabelecem o que requer ações mais curativas ou de recuperação, em detrimento da prevenção. O resgate da condição de saúde prejudicada na pessoa/ser humano idoso passa a ser o hospital, ambiente complexo, envolto por inovações tecnológicas surpreendentes e desconhecidas, e de vital importância no contexto de recuperação da saúde do ser que envelhece e adoece. Como exercitar uma prática de cuidado de enfermagem que contribua para um desempenho mais humanizado diante de inovações e aparatos tecnológicos que monitora a vida do cidadão em ambientes hospitalares cada vez mais avançados?

Por outro lado, a estrutura hospitalar sustentada por normatizações e rotinas, o biopoder exercido pelos profissionais da saúde fruto dessa hierarquização estrutural, pode dificultar a possibilidade de espaço para o exercício da autonomia pelo cidadão, ser humano/idoso hospitalizado. Como o poder decisório das pessoas, trabalhadores, pacientes/pessoa humana doente e gestores, podem ultrapassar as barreiras ou limites das estruturas formais das instituições hospitalares e tornar-se mais flexível diante de desempenhos e competências diversificadas e ações que necessitam ser otimizadas e concatenadas?

As relações de cuidado exigem sustentabilidade legal e moral. $\mathrm{O}$ respeito à dignidade do idoso hospitalizado confirma sua autonomia e deve ser embasado num princípio ético de valorização da vontade humana. Como exercitar uma prática de cuidado com reciprocidade, solidariedade e respeito ao direito do ser humano na tomada de decisão sobre a sua doença e ao seu corpo diante de estruturas hierárquicas formais, com normas e rotinas tradicionais? Como sensibilizar as pessoas na ambiente hospitalar para um olhar diferenciado sobre as debilidades ou dependências de cuidado das pessoas idosas internadas e suas possibilidades de participar na tomada de decisão sobre ações que desconhecem ou não mais acompanham a evolução da sua complexidade técnica-científica? 
Como sensibilizar os estudantes, futuros profissionais da saúde, para o exercício pleno do cuidado humanizado?

Os profissionais de enfermagem através do cuidado de enfermagem no ambiente hospitalar podem contribuir significativamente para a construção da autonomia e participação das pessoas idosas na tomada de decisão sobre suas necessidades de atenção ou cuidado à sua saúde.

O resgate da ética numa perspectiva de envelhecimento digno perpassa por diversas discussões na sociedade que envelhece e, especificamente, entre os profissionais de saúde que atuam nas instituições hospitalares.

A atitude de cuidado requer respeito ao direito do paciente na tomada de decisão sobre a sua doença e o seu corpo, exercitando o respeito à dignidade humana como prática no cotidiano hospitalar.

Eis o grande desafio: construir a civilidade humana, exercitando um cuidado de enfermagem humano, científico e tecnologicamente avançado, respeitando a autonomia do cidadão e o direito e respeito sobre si, na sua relação de autonomia e dependência. Será possível?

\section{REFERÊNCIAS}

1. IBGE. Censo Demográfico. Brasil, 2000. Rio de Janeiro, IBGE, 2000.

2. Rio Grande do Sul. Secretaria Estadual de Saúde. Centro Estadual de Vigilância em Saúde. A saúde da população do estado do Rio Grande do Sul. Porto Alegre: CEVS, 2006.

3. Alves, LC, Leimann BCQ, Vasconcelos MEL, Carvalho MS, Vasconcelos AGG, Fonseca TCO et al. A influência das doenças crônicas na capacidade funcional dos idosos do Município de São Paulo, Brasil. Cad. de Saúde Pública, 2007;23(8):1924-30, ago,

4. Brasil. Ministério da Previdência e Assistência Social (BR). Seminário Internacional de Envelhecimento Populacional, 2002, Brasília: Anais do Ministério de Previdência e Assistência à saúde. Brasília, 2002.

5. Nightingale, F. Notas sobre enfermagem: o que é e o que não é. São Paulo: Cortez, 1989.

6. Schwanke, CHA, Cruz, IBM. Ética do cuidado na sociedade que envelhece: contribuições ao debate. In: Clotet; J, Feijó; A, Gerhardt, MO, organizador. Bioética- uma visão panorâmica. Porto Alegre: EDIPUCRS, 2005, v.1, p. 267-75
7. Aghich, JG. Dependência e autonomia na velhice. Um modelo ético para o cuidado de longo prazo. São Paulo: Loyola; Centro Universitário São Camilo, 2008.

8. Beauchamp, TL, Childress, JF. Princípios de ética biomédica.São Paulo: Loyola, 2002.

9. Brasil. Portaria $n^{\circ} 2.528$ de 19 de outubro de 2006. Aprova a Política Nacional de Saúde da Pessoa Idosa. Diário Oficial da União 2006 out 26;1:142-5.

10. Sgreccia, E. Manual de Bioética I. Fundamentos e ética biomédica. São Paulo: Loyola, 2002.

11. Campi S. O valor intrínseco da vida e a autonomia: reflexões sobre a eutanásia. [Dissertação] Florianópolis (SC): Universidade Federal de Santa Catarina, 2004.

12. Goldim, JR. Rompendo os limites entre a ética e a ciência. Porto Alegre: Episteme, 2000.

13. Kant, I. Fundamentação da metafísica dos costumes. Rio de Janeiro: Companhia Editora Nacional, 1996.

14. Bushatsky, M. Princípio da Autonomia: do conhecimento à práxis na oncologia pediátrica. [Dissertação] Recife (PE): Universidade Federal de Pernambuco, 2005. 